\title{
Far-IR Absorption of Neutral Polycyclic Aromatic Hydrocarbons (PAHs): Light on the Mechanism of IR-UV Ion Dip Spectroscopy
}

Alexander K. Lemmens, Daniël B. Rap, Johannes M. M. Thunnissen, Sébastien Gruet, Amanda L. Steber, Sanjana Panchagnula, Alexander G. G. M. Tielens, Melanie Schnell, Wybren Jan Buma,* and Anouk M. Rijs*

Cite This: J. Phys. Chem. Lett. 2020, 11, 8997-9002

Read Online

ACCESS | 네 Metrics \& More | 回 Article Recommendations | st Supporting Information

ABSTRACT: Gas-phase IR-UV double-resonance laser spectroscopy is an IR absorption technique that bridges the gap between experimental IR spectroscopy and theory. The IR experiments are used to directly evaluate predicted frequencies and potential energy surfaces as well as to probe the structure of isolated molecules. However, a detailed understanding of the underlying mechanisms is, especially in the far-IR regime, still far from complete, even though this is crucial for properly interpreting the recorded IR absorption spectra. Here, events occurring upon excitation to vibrational levels of polycyclic aromatic hydrocarbons by far-IR radiation from the FELIX free electron laser are followed using resonance-enhanced multiphoton ionization spectroscopy. These studies provide detailed insight into how ladder climbing and anharmonicity influence IR-UV spectroscopy and therefore the resulting IR signatures in the far-IR region. Moreover, the potential energy surfaces of these low-frequency delocalized modes are investigated and shown to have a strong harmonic character.

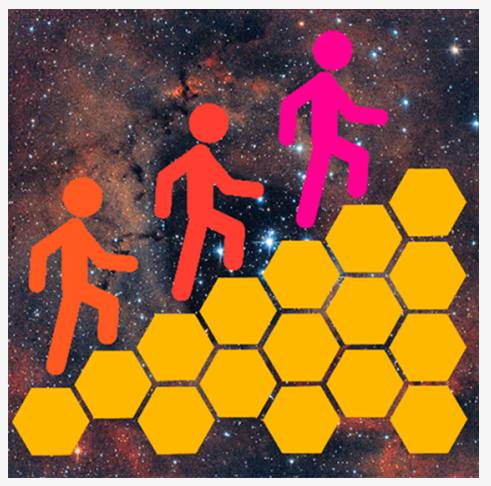

Thfrared absorption spectroscopy has proven to be a 1 powerful technique for characterizing and monitoring the physical and chemical properties of molecules. The technique provides vibrational fingerprints that give direct access to their electronic and conformational structure. At the same time, they are also essential for obtaining insight into their thermochemistry and reaction dynamics. At the core of these applications is a full and detailed understanding of vibrations in terms of nuclear motions and the characteristics of the multidimensional electronic potential energy surface on which these motions take place. This is most effectively realized by comparing experimentally measured quantities with theoretical predictions. Such comparisons provide on one hand the means to assign an experimental spectrum but, equally importantly, are also key to assessing theoretical methods. High-resolution spectroscopy on isolated molecules plays in this respect an important role as it is the most direct means for making such comparisons. IR-UV ion dip spectroscopy is the ideal method for obtaining high-resolution, mass- and conformer-selective IR spectra. ${ }^{1}$ In an astronomical context, IR-UV spectroscopy has proven to be indispensable for providing accurate band positions for relevant molecules such as polycyclic aromatic hydrocarbons (PAHs) and for evaluating various theoretical methods that aim to incorporate anharmonicity. ${ }^{2-8}$ More widely, it is being used for the structural characterization of neutral and isolated molecules that range from peptides and DNA bases to molecular machines. ${ }^{1,9-13}$

The comparison between experiment and theory relies on not only the band positions but also their intensities. The latter are of particular importance within the framework of astrophysics where relative and absolute cross sections are used to determine the composition and abundance of molecular species in the universe. ${ }^{14}$ To relate IR-UV ion dips to absolute absorption cross sections, a detailed understanding of the IR-UV ion dip mechanism is essential. Although the general principles of the method are well understood, detailed insight into the underlying processes is to a large extent lacking. The far-IR region is of special interest because it is less congested $^{15,16}$ than the mid- or near-IR regions, and the emission from interstellar space in the far-IR region is inherently less broad. ${ }^{17}$ Moreover, the low-frequency modes of PAHs involve their global structure and thereby provide diagnostic fingerprints of their size and topology.

IR-UV ion dip spectroscopy is based on the change of the vibrational population in the electronic ground state of a molecule by IR absorption, which is probed by resonanceenhanced multiphoton ionization (REMPI) spectroscopy. Resonant IR excitation leads to a decrease in the magnitude of the REMPI signal due to the difference in vibrational frequencies in the electronic ground and excited state, and/or

Received: September 6, 2020

Accepted: September 30, 2020

Published: October 9, 2020 
the change in the overlap of the vibrational wave functions. ${ }^{18}$ Often, a decrease of $>50 \%$ is observed, which implies that the IR absorption step cannot simply be described with a two-level model consisting of the vibrationally ground and excited state. Instead, different processes involving more than two states, such as IR-induced fragmentation or intramolecular vibrational energy redistribution (IVR), must occur within the time scale of these experiments. ${ }^{19,20}$ More insight into the IR-UV depletion mechanism can be obtained by probing the molecule after IR absorption and determining the vibrational state(s) that is populated. This can in principle be done by recording REMPI excitation spectra after IR excitation. ${ }^{18,21-24}$ Such an approach is especially attractive for far-IR vibrational modes where excitation localizes the population in a few eigenstates, whereas in the case of near-IR excitation, statistical inhomogeneous broadening by IVR impedes a straightforward interpretation of the UV excitation spectrum. ${ }^{18}$

Here we report far-IR studies on naphthalene, anthracene, phenanthrene, acenaphthene, acenaphthene- $d_{10}$, chrysene, pyrene, and benzo[ghi]perylene, all of which are prototypical PAHs that feature various topological aspects of importance (see Figure SI.1). The far-IR region has so far remained out of reach for PAHs under astronomically relevant conditions, that is, as isolated gas-phase molecules and at low temperatures. Table SI.1 summarizes the experimental IR frequencies found for each molecule in the region of $80-250 \mathrm{~cm}^{-1}$ together with the calculated frequencies in this region.

Generally, delocalized, low-frequency vibrational modes of aromatic rings, such as ring puckering and other largeamplitude motions, are being termed anharmonic, ${ }^{25-29}$ but from an experimental point of view, this is far from clear. In our experiments, we probe not only the depopulation of the vibrational ground state but also the population of distinct lowenergy vibrationally excited states. Our studies, therefore, enable us to obtain insight into the mechanism of IR-UV ion dip spectroscopy and to determine the (an)harmonic character of delocalized low-frequency vibrational modes of PAHs.

The black traces in Figure 1 show the UV excitation spectrum near the $0_{0}^{0}$ origin band of acenaphthene- $d_{10}$, while the red traces correspond to REMPI spectra taken after resonant IR excitation of a mid-IR band at $1604 \mathrm{~cm}^{-1}$ (CC inplane vibration) and a far-IR band at $189 \mathrm{~cm}^{-1}$ (drumhead motion). Excitation in the mid-IR region causes depopulation of the ground state and leads to statistical inhomogeneous broadening of the origin band due to the high density of states at this energy. ${ }^{18} \mathrm{IR}$ absorption in this region thus results in depletion at the band origin frequency (see Figure SI.2) and a smoothly varying IR ion gain (IRIG) $)^{23,24}$ at frequencies other than the band origin. In contrast, in the far-IR excitation experiment, a low-frequency vibrational state is populated at an energy where the density of states is much lower. As a result, IR excitation produces a vibrational population distribution over only a few eigenstates, leading to resolved features in the UV excitation spectrum that probes this distribution. In the following, we will first assign these resolvable IRIG bands and then discuss the implications of these assignments on the mechanism of far-IR-UV ion dip spectroscopy. Finally, we will evaluate the (an)harmonic properties of the low-frequency modes of the studied PAHs on the basis of the observed spectral features.

The appearance of resonant transitions in the ion gain spectrum (Figure 1b) at frequencies lower than the origin band, after excitation of $\nu_{3}$ at $189 \mathrm{~cm}^{-1}$, indicates that distinct
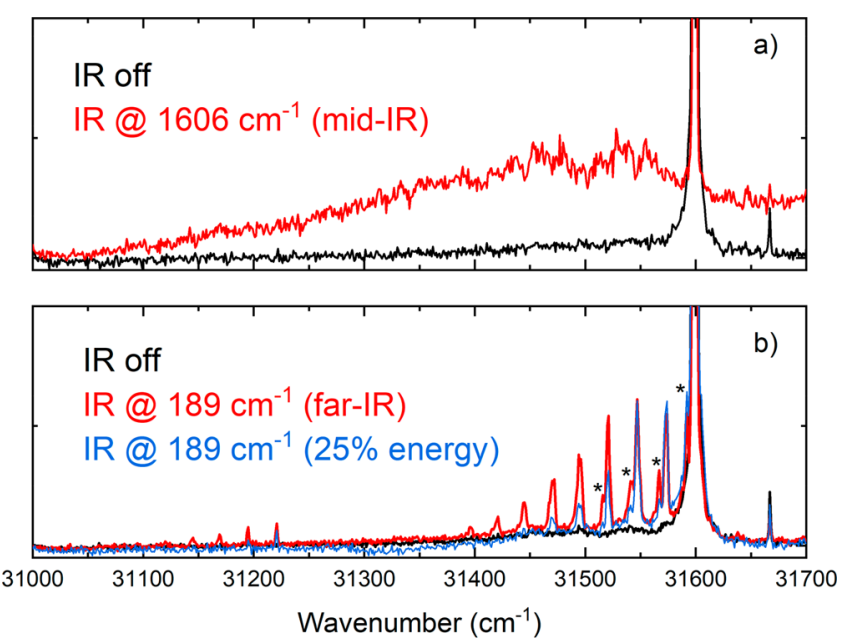

Figure 1. Experimental REMPI excitation spectrum (black traces) of the origin band of acenaphthene- $d_{10}$ at $31599 \mathrm{~cm}^{-1}$ recorded in the absence of IR excitation. The colored traces show spectra with the IR laser FELIX preceding the REMPI process at a resonant frequency of (a) $1606 \mathrm{~cm}^{-1}$ (pulse energy of $13 \mathrm{~mJ}$, red) or (b) $189 \mathrm{~cm}^{-1}$ [6 mJ (red) and $1.5 \mathrm{~mJ}$ (blue), red and blue traces scaled such that the $\left(\nu_{3}\right)_{v^{\prime \prime}=2}^{v^{\prime}=2}$ bands have the same intensity]. In both cases, there is a decrease [ion dip (see Figure SI.2)] at the origin transition and an increase (ion gain) at frequencies other than the origin after IR absorption. The unresolved gain in panel a reflects the outcome of statistical inhomogeneous broadening, while panel $\mathrm{b}$ shows distinct bands indicating that vibrational excitation is restricted to only a few eigenstates.

vibrational levels in the electronic ground state are populated after far-IR excitation. The ion gain bands show a regular spacing of $25.5 \mathrm{~cm}^{-1}$, which necessarily must be associated with $\left(\nu_{3}\right)_{v^{\prime \prime}=n}^{v^{\prime}=n}$ transitions because $25.5 \mathrm{~cm}^{-1}$ is lower than the lowest-frequency vibrational mode of acenaphthene- $d_{10}$. Such a regular spacing is possible only if the potential energy surface in the electronic ground and excited state along the normal mode $\nu_{i}$ is highly harmonic. Indeed, we find from the variation in band spacings an anharmonicity of $\sim 0.1 \%$.

A schematic energy diagram with the possible IR and UV excitation routes is shown in Figure 2. The ladder-climbing pathway displayed in Figure $2 \mathrm{a}$ results in the population of higher vibrational levels of the harmonic vibrational mode at the IR frequency for which the fundamental transition is excited (possible deviation of $1 \%$ resulting from the full width at half-maximum of FELIX). Vibrational overlap considerations dictate that UV transitions will predominantly take place between vibrational levels with the same vibrational quantum number in the electronic ground and excited state. Because the frequencies of vibrational modes in these two states are different, with typically lower frequencies in the excited state, the $\left(\nu_{3}\right)_{v^{\prime \prime}=n}^{v^{\prime}=n}$ transitions are observed as a regular red-shifted progression with a spacing corresponding to the difference in the vibrational energy of mode $\nu_{i}$ in the two electronic states. Such a ladder-climbing pathway was considered in the far-IR study of tryptamine, but the presence of multiple conformers precluded an unambiguous assignment of the observed transitions, unlike what is possible in the study presented here. $^{22}$

Figure 1 shows that the IR pulse energy has a marked influence on the REMPI spectrum. First, we observe that with a lower pulse energy (blue trace) higher vibrational quanta 


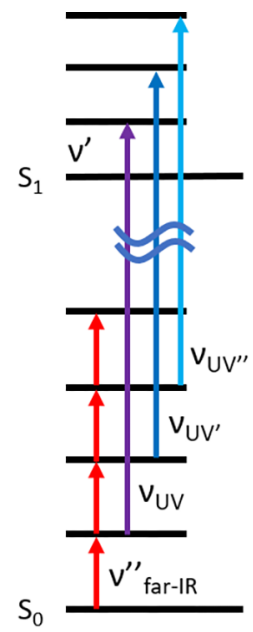

a) IRIG harmonic regular progression $\left(v^{\prime \prime}>v^{\prime}\right)$

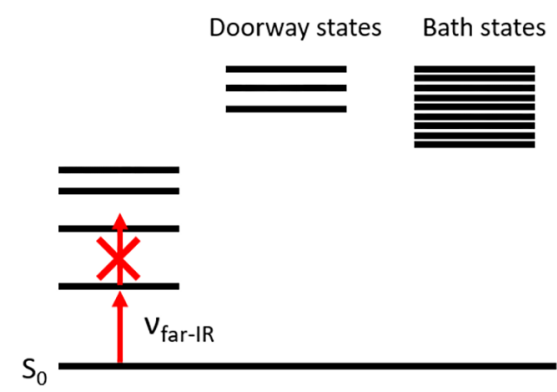

b) 'restricted' depopulation of ground state

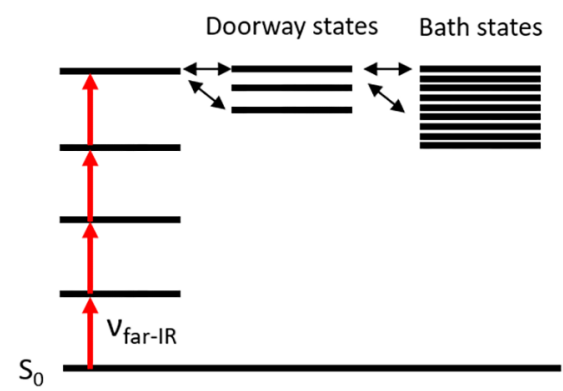

c) 'unrestricted’ depopulation of ground state

Figure 2. (a) Schematic representation of ladder climbing leading to a regular red-shifted progression in IRIG spectra. (b) "Restricted" absorption in an anharmonic potential with nonequidistant vibrational levels, which results in IVR for low-frequency vibrational modes being largely inhibited. (c) "Unrestricted" depopulation of the ground state in a harmonic potential with equidistant vibrational levels where ladder climbing in lowfrequency vibrational modes allows access to otherwise nonaccessible IVR pathways.

levels are not reached. Second, the red trace taken at higher pulse energies shows bands marked with an asterisk that are not (or hardly) present in the blue trace. These bands are associated with transitions involving the $\left(\nu_{2}\right)_{v^{\prime \prime}=1}^{v^{\prime}=1}$ transition. Their presence implies a redistribution of vibrational energy to the $\left(\nu_{2}\right)_{v^{\prime \prime}=1}$ level that clearly occurs only if levels of $\nu_{3}$ are reached with sufficiently high quantum numbers. Similar redistributions are observed at other IR frequencies and for the other molecules we have studied (see Figure 4). Generally, we find that at very low IR frequencies, we can still assign quite reliably transitions occurring upon IVR, but for higherfrequency transitions, the number of possibilities becomes too large for unambiguous assignment.

Ladder climbing in harmonic vibrational modes has important consequences for the interpretation of IR ion dip intensities. In the case of anharmonic modes, only one photon can be absorbed (Figure 2b). For low-frequency modes, the density of states at the accessed vibrational energy is so low that IVR is not possible, resulting in a maximum depopulation of $50 \%$ of the ground state. Ladder climbing followed by IVR from higher vibrational levels (Figure 2c) allows for multiple IR photon absorption and depopulation of the ground state by $>50 \%$. The (an)harmonic character of a vibrational mode thus strongly determines the amount of depopulation of the ground state and, thereby, the observed IR ion dip intensity.

The pathways described above imply that REMPI spectra obtained after far-IR excitation should depend critically on the IR laser pulse energy. On one hand, the harmonic ladder is climbed to relatively low vibrational quantum numbers at low energies and IVR is restricted. At higher pulse energies, on the other hand, vibrational levels with higher quantum numbers are accessed and IVR is enhanced. Our experiments confirm this: at a low IR laser pulse energy (Figure 3d, red trace), distinct, narrow transitions are observed and IVR-induced broadening is limited. As we move to higher pulse energies, these features disappear as an IVR threshold is reached and only a broadened IR-induced REMPI feature remains (Figure 3a, purple trace).

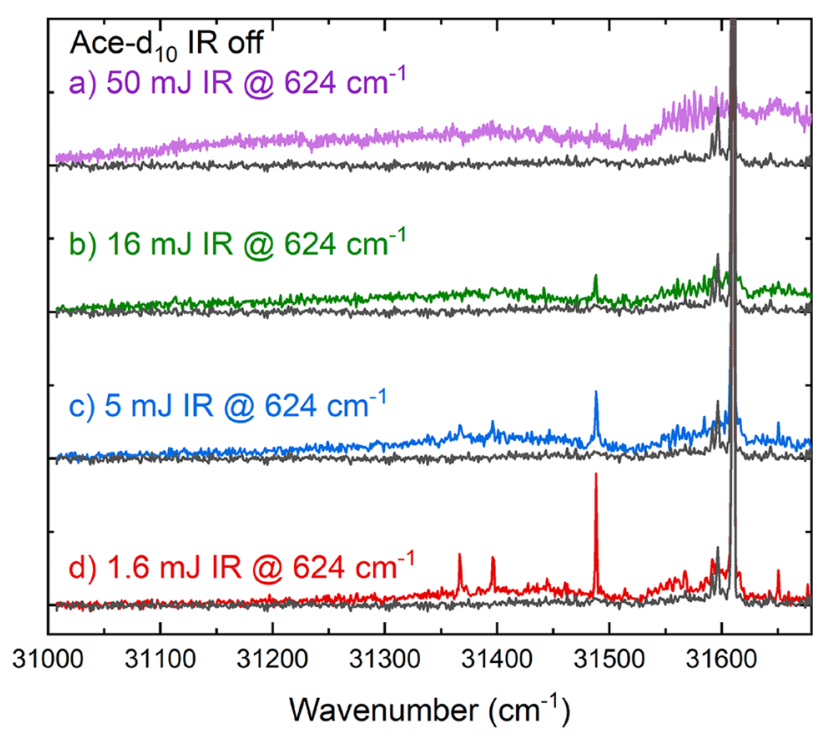

Figure 3. REMPI spectra near the origin region of acenaphthene- $d_{10}$ at an IR excitation frequency of $624 \mathrm{~cm}^{-1}$. The IR laser pulse energies used were approximately (a) $50 \mathrm{~mJ}$ (purple), (b) $16 \mathrm{~mJ}$ (green), (c) 5 $\mathrm{mJ}$ (blue), and (d) $1.6 \mathrm{~mJ}$ (red). The black trace in each plot shows the REMPI excitation spectrum without IR excitation and serves as a reference. Unscaled REMPI spectra are provided in Figure SI.3.

Another important consequence of this power dependence is that one should be cautious when interpreting signal intensities in IRIG spectra because a lower IR pulse energy might very well result in a stronger IRIG signal at particular UV probe frequencies. IRIG signals are, thus, not necessarily related to IR cross sections in a 1:1 manner. Moreover, normally an exponential relation between laser power and IR depletion is assumed, but this model is based on the assumption of a fast irreversible IVR process without a threshold. This may clearly fail under conditions such as those used in our experiments.

Figure 4 displays an overview of REMPI spectra measured near the origin transition to the lowest electronically excited singlet state of selected PAHs with (color) and without (black) 


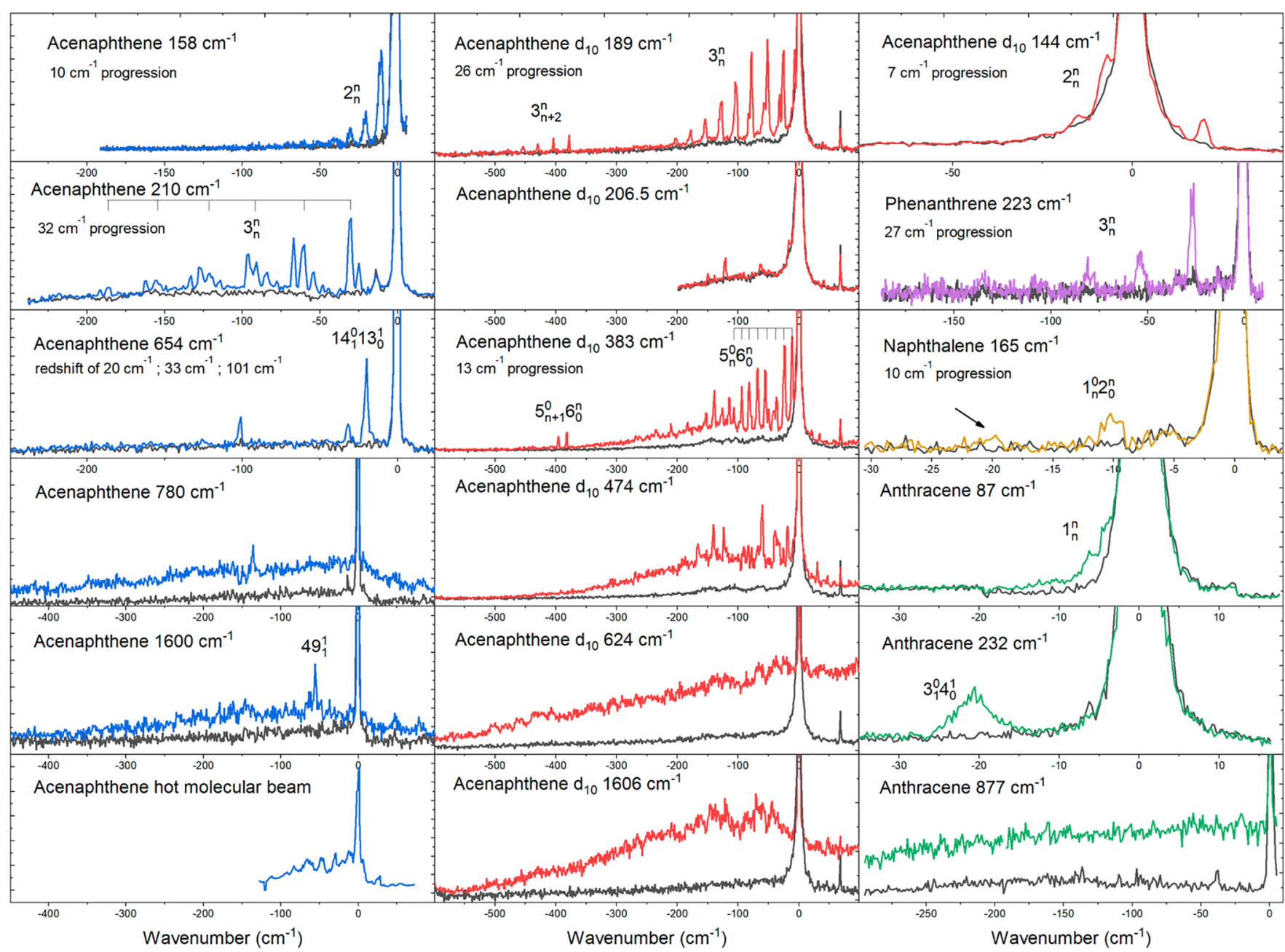

Figure 4. Experimental REMPI spectra of naphthalene, acenaphthene, acenaphthene- $d_{10}$, anthracene, and phenanthrene (black traces) near the origin bands (set to zero). The colored traces show REMPI excitation spectra with the IR laser at the resonant IR frequency indicated within each subplot preceding REMPI probing. Full unscaled spectra are provided in Figure SI.2.

IR pre-excitation. The IR frequencies range from the far-IR to mid-IR (for unscaled spectra, see Figure SI.2). For IR excitation up to $600 \mathrm{~cm}^{-1}$, regular progressions are observed, while excitation at higher frequencies leads to broadened spectra. The vibrational modes are either out-of-plane butterfly or drumhead motions, except for those of acenaphthene- $d_{10}$ $\left(383 \mathrm{~cm}^{-1}\right)$ and anthracene $\left(232 \mathrm{~cm}^{-1}\right)$, which are in-plane scissoring modes. A full overview of the transitions that have been assigned on the basis of quantum chemical calculations in the electronic ground and excited states of the pertinent compound is provided in Table SI.2. Secondary progressions associated with transitions involving a decrease in the vibrational quantum number are visible for acenaphthene- $d_{10}$ upon excitation with IR frequencies of 189 and $383 \mathrm{~cm}^{-1}$. In the case of $189 \mathrm{~cm}^{-1}$ excitation, a second progression is shifted by exactly twice the fundamental frequency, while for the 383 $\mathrm{cm}^{-1}$ excitation, the second progression is shifted by exactly the fundamental frequency. The assignment of the progression of naphthalene to a transition between two different vibrational mode numbers is justified by the fact that these modes are the same normal modes and, therefore, have the largest vibrational overlap. A similar situation exists for the $232 \mathrm{~cm}^{-1}$ IR-pumped REMPI spectrum of anthracene. In some IR ion gain spectra, the red-shifted bands consist of a doublet or series of bands, e.g., for acenaphthene- $d_{10}$ with $189 \mathrm{~cm}^{-1}$ and acenaphthene with $210 \mathrm{~cm}^{-1}$ IR excitation. We tentatively assign these bands to result from lower-frequency $\left(\nu_{i}\right)_{v^{\prime \prime}=1}^{v^{\prime}=1}$ transitions that become observable after IVR to a lower-frequency mode $\nu_{i}$.

Figure 4 (bottom, left) also displays a "hot" molecular beam REMPI spectrum of acenaphthene that illustrates the difference between the population of vibrational states at a higher temperature $^{30}$ and the population of vibrational states due to resonant IR excitation. A clear difference in structure is observed between the hot bands in the "hot" spectrum and the IR ion gain features in the REMPI spectra obtained with 1600 or $780 \mathrm{~cm}^{-1}$ excitation. Compared to the $1600 \mathrm{~cm}^{-1}$ IRIG spectrum, there is relatively more intensity close to the band origin in the "hot" spectrum. The $780 \mathrm{~cm}^{-1}$ gain spectrum shows a broadening of the band origin to both the red and blue side of the band origin, whereas the "hot" spectrum shows the most intensity on the red side. Resonant vibrational excitation cannot simply be seen as creating a Boltzmann distribution of vibrational energy. Even well within the mid-IR range, a notable difference in structure is observed in the IR ion gain, which becomes clear when the 624 and $1606 \mathrm{~cm}^{-1}$ excitation REMPI spectra of acenaphthene- $d_{10}$ are compared. Similarly, this can be seen in the 780 and $1600 \mathrm{~cm}^{-1}$ excitation REMPI spectra of acenaphthene. These observations indicate that the 
redistribution of vibrational energy follows quite different pathways after excitation at 780 or $1600 \mathrm{~cm}^{-1}$.

An interesting case arises when the vibrational frequencies of the ground and electronically excited states are very similar; the normally observed red-shift in the ion gain REMPI spectra is no longer resolvable. This occurs partly in the case of the IR excitation of acenaphthene- $d_{10}$ at $144 \mathrm{~cm}^{-1}$. REMPI spectra taken after IR excitation show a red shift of $\sim 7 \mathrm{~cm}^{-1}$, partly falling within the width of the origin transition and impeding optimal detection of depletion. This observation is in particular important in the context of studies of large PAHs as these PAHs undergo only a small structural change upon electronic excitation and have similar vibrational energies in the electronic ground and excited state. To be able to probe the effect of IR absorption, one relies on a change in FranckCondon factors upon excitation or of the ionization cross section from the electronically excited state.

REMPI spectra obtained after IR excitation of specific vibrational levels in the far-IR and mid-IR regions have provided unique insight into the mechanisms of IR-UV ion dip spectroscopy in the different frequency regimes. At low IR frequencies, vibronic transitions from distinct vibrational levels have been observed that unambiguously prove ladder climbing occurs. This is an important conclusion as it means that many more transitions can contribute to the depletion of the vibration-less ground state than what has been assumed to date. Moreover, vibrational energy redistribution pathways become available that previously were not taken into account. The IVR threshold that, depending on the IR photon energy, can or cannot be reached can introduce nonlinearity into the intensity of IR ion dip absorption bands. This conclusion provides a gratifying explanation for puzzling observations from the past, in which low-frequency modes appeared with a much higher intensity in IR-UV ion dip spectra than predicted by theoretical calculations. At higher frequencies, vibrational energy redistribution readily occurs, which, in combination with differences in vibrational frequencies in the electronic ground and excited states, leads to a reasonably smooth ion gain feature.

The observation of ladder climbing in low-frequency vibrational modes is surprising because it has been assumed that these modes would be quite anharmonic as calculated anharmonic force constants are relatively large. To calibrate (an)harmonic theory for the PAHs in the far-IR range, we have compared experimental IR frequencies obtained in this work with theoretical predictions of harmonic and anharmonic frequencies (Figure SI.4). As expected from the IRIG results, the scaled harmonic frequency predictions are at least as accurate as anharmonic frequencies. Contrary to a priori expectations, the low-frequency modes are very harmonic (some with a deviation of only $0.1 \%$ ), which is, from an astronomical point of view, positive news because harmonic models are often used to interpret interstellar infrared emission features. They can thus be used to accurately predict far-IR frequencies.

\section{EXPERIMENTAL SECTION}

The experiments were performed at the FELIX laboratory in The Netherlands using a pulsed molecular beam time-of-flight mass spectrometer and are fully described in the Supporting Information. ${ }^{31}$ The mass-selective IR spectra of the selected PAHs were recorded by IR-UV ion dip spectroscopy using the free electron laser FELIX. Subsequently, their IR ion gain spectra were obtained by fixing the IR laser at the selected IR absorption, while scanning the UV laser. The assignments are supported by harmonic IR frequency calculations performed using density functional theory at the B3LYP/6-31G* level of theory in the Gaussian 16 program package. ${ }^{32,33}$ Anharmonic frequency calculations are performed using the same package at the B3LYP/N07D level of theory.

\section{ASSOCIATED CONTENT}

\section{Supporting Information}

The Supporting Information is available free of charge at https://pubs.acs.org/doi/10.1021/acs.jpclett.0c02714.

Supplementary figures and more extended experimental method (PDF)

Line list of calculated vibrational frequencies (XLSX)

\section{AUTHOR INFORMATION}

\section{Corresponding Authors}

Wybren Jan Buma - University of Amsterdam, 1098 XH Amsterdam, The Netherlands; Radboud University, Institute for Molecules and Materials, FELIX Laboratory, 6525 ED Nijmegen, The Netherlands; (1) orcid.org/0000-0002-12658016; Email: w.j.buma@uva.nl

Anouk M. Rijs - Radboud University, Institute for Molecules and Materials, FELIX Laboratory, 6525 ED Nijmegen, The Netherlands; ○ orcid.org/0000-0002-7446-9907; Email: a.m.rijs@vu.nl

\section{Authors}

Alexander K. Lemmens - Radboud University, Institute for Molecules and Materials, FELIX Laboratory, 6525 ED Nijmegen, The Netherlands; University of Amsterdam, 1098 XH Amsterdam, The Netherlands

Daniël B. Rap - Radboud University, Institute for Molecules and Materials, FELIX Laboratory, 6525 ED Nijmegen, The Netherlands

Johannes M. M. Thunnissen - Radboud University, Institute for Molecules and Materials, FELIX Laboratory, 6525 ED Nijmegen, The Netherlands

Sébastien Gruet - Deutsches Elektronen-Synchrotron, D-22607 Hamburg, Germany; Institut für Physikalische Chemie, Christian-Albrechts-Universität zu Kiel, D-24118 Kiel, Germany

Amanda L. Steber - Deutsches Elektronen-Synchrotron, D22607 Hamburg, Germany; Institut für Physikalische Chemie, Christian-Albrechts-Universität zu Kiel, D-24118 Kiel,

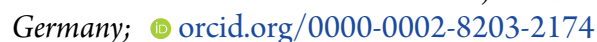

Sanjana Panchagnula - Leiden Observatory, Leiden University, 2333 CA Leiden, The Netherlands

Alexander G. G. M. Tielens - Leiden Observatory, Leiden University, 2333 CA Leiden, The Netherlands

Melanie Schnell - Deutsches Elektronen-Synchrotron, D-22607 Hamburg, Germany; Institut für Physikalische Chemie, Christian-Albrechts-Universität zu Kiel, D-24118 Kiel, Germany; 이이이.org/0000-0001-7801-7134

Complete contact information is available at:

https://pubs.acs.org/10.1021/acs.jpclett.0c02714

\section{Notes}

The authors declare no competing financial interest. 


\section{ACKNOWLEDGMENTS}

The authors thank Prof. Dr. Michael Schmitt for his useful discussions and help during this project. Also, the authors thank the FELIX laboratory team for their experimental assistance and scientific support and acknowledge The Netherlands Organization for Scientific Research (NWO) for the support of the FELIX Laboratory and SURFsara for their computational resources. This work was supported by The Netherlands Organization for Scientific Research (NWO) and is part of the Dutch Astrochemistry Network (DAN) II (Project 648.000.029).

\section{REFERENCES}

(1) Schwing, K.; Gerhards, M. Investigations on Isolated Peptides by Combined IR/UV Spectroscopy in a Molecular Beam - Structure, Aggregation, Solvation and Molecular Recognition. Int. Rev. Phys. Chem. 2016, 35 (4), 569-677.

(2) Maltseva, E.; Mackie, C. J.; Candian, A.; Petrignani, A.; Huang, X.; Lee, T. J.; Tielens, A. G. G. M.; Oomens, J.; Buma, W. J. HighResolution IR Absorption Spectroscopy of Polycyclic Aromatic Hydrocarbons in the $3 \mu \mathrm{m}$ Region: Role of Hydrogenation and Alkylation. Astron. Astrophys. 2018, 610, A65.

(3) Piest, H.; von Helden, G.; Meijer, G. Infrared Spectroscopy of Jet-Cooled Cationic Polyaromatic Hydrocarbons: Naphthalene. Astrophys. J. 1999, 520 (1), L75-L78.

(4) Roithová, J.; Jašík, J.; Del Pozo Mellado, J. J.; Gerlich, D. Electronic Spectra of Ions of Astrochemical Interest: From Fast Overview Spectra to High Resolution. Faraday Discuss. 2019, 217, 98-113.

(5) Basire, M.; Parneix, P.; Calvo, F. Finite-Temperature IR Spectroscopy of Polyatomic Molecules: A Theoretical Assessment of Scaling Factors. J. Phys. Chem. A 2010, 114 (9), 3139-3146.

(6) Candian, A.; Mackie, C. J. Anharmonic Interstellar PAH Molecules. Int. J. Quantum Chem. 2017, 117 (2), 146-150.

(7) Oomens, J.; Tielens, a. G. G. M.; Sartakov, B. G.; von Helden, G.; Meijer, G. Laboratory Infrared Spectroscopy of Cationic Polycyclic Aromatic Hydrocarbon Molecules. Astrophys. J. 2003, 591 (2), 968-985.

(8) Bloino, J. A. VPT2 Route to Near-Infrared Spectroscopy: The Role of Mechanical and Electrical Anharmonicity. J. Phys. Chem. A 2015, 119 (21), 5269-5287.

(9) Gloaguen, E.; Mons, M. Isolated Neutral Peptides. Top. Curr. Chem. 2014, 364, 225-270.

(10) de Vries, M. S.; Hobza, P. Gas-Phase Spectroscopy of Biomolecular Building Blocks. Annu. Rev. Phys. Chem. 2007, 58 (1), 585-612.

(11) Bakels, S.; Gaigeot, M. P.; Rijs, A. M. Gas-Phase Infrared Spectroscopy of Neutral Peptides: Insights from the Far-IR and THz Domain. Chem. Rev. 2020, 120, 3233.

(12) Ishiuchi, S. I.; Yamada, K.; Oba, H.; Wako, H.; Fujii, M. Gas Phase Ultraviolet and Infrared Spectroscopy on a Partial Peptide of $\beta_{2}$-Adrenoceptor SIVSF- $\mathrm{NH}_{2}$ by a Laser Desorption Supersonic Jet Technique. Phys. Chem. Chem. Phys. 2016, 18 (33), 23277-23284.

(13) Pribble, R. N.; Zwier, T. S. Size-Specific Infrared Spectra of Benzene- $\left(\mathrm{H}_{2} \mathrm{O}\right)_{n}$ Clusters $(\mathrm{n}=1$ through 7$)$ : Evidence for Noncyclic $\left(\mathrm{H}_{2} \mathrm{O}\right)_{\mathrm{n}}$ Structures. Science 1994, 265 (5168), 75-79.

(14) Bauschlicher, C. W.; Ricca, A.; Boersma, C.; Allamandola, L. J. The NASA Ames PAH IR Spectroscopic Database: Computational Version 3.00 with Updated Content and the Introduction of Multiple Scaling Factors. Astrophys. J. Suppl. Ser. 2018, 234 (2), 32.

(15) Mulas, G.; Malloci, G.; Joblin, C.; Toublanc, D. Diagnostics for Specific PAHs in the Far-IR: Searching Neutral Naphthalene and Anthracene in the Red Rectangle. Astron. Astrophys. 2006, 456, 161169.

(16) Boersma, C.; Bauschlicher, C. W.; Allamandola, L. J.; Ricca, A.; Peeters, E.; Tielens, A. G. G. M. The 15-20 Mm PAH Emission
Features: Probes of Individual PAHs? Astron. Astrophys. 2010, 511, A32.

(17) Mackie, C. J.; Chen, T.; Candian, A.; Lee, T. J.; Tielens, A. G. G. M. Fully Anharmonic Infrared Cascade Spectra of Polycyclic Aromatic Hydrocarbons. J. Chem. Phys. 2018, 149 (13), 134302.

(18) Nagornova, N. S.; Rizzo, T. R.; Boyarkin, O. V. Exploring the Mechanism of IR-UV Double-Resonance for Quantitative Spectroscopy of Protonated Polypeptides and Proteins. Angew. Chem., Int. Ed. 2013, 52 (23), 6002-6005.

(19) Coveleskie, R. A.; Dolson, D. A.; Parmenter, C. S. A Direct View of Intramolecular Vibrational Redistribution in S1p-Difluorobenzene. J. Chem. Phys. 1980, 72 (10), 5774-5775.

(20) Oomens, J.; Sartakov, B. G.; Meijer, G.; von Helden, G. GasPhase Infrared Multiple Photon Dissociation Spectroscopy of MassSelected Molecular Ions. Int. J. Mass Spectrom. 2006, 254 (1-2), 119.

(21) Yamada, Y.; Mikami, N.; Ebata, T. Real-Time Detection of Doorway States in the Intramolecular Vibrational Energy Redistribution of the OH/OD Stretch Vibration of Phenol. J. Chem. Phys. 2004, 121 (23), 11530-11534.

(22) Schmitt, M.; Spiering, F.; Zhaunerchyk, V.; Jongma, R. T.; Jaeqx, S.; Rijs, A. M.; van der Zande, W. J. Far-Infrared Spectra of the Tryptamine A Conformer by IR-UV Ion Gain Spectroscopy. Phys. Chem. Chem. Phys. 2016, 18 (47), 32116-32124.

(23) Dean, J. C.; Buchanan, E. G.; James, W. H.; Gutberlet, A.; Biswas, B.; Ramachandran, P. V.; Zwier, T. S. Conformation-Specific Spectroscopy and Populations of Diastereomers of a Model Monolignol Derivative: Chiral Effects in a Triol Chain. J. Phys. Chem. A 2011, 115 (30), 8464-8478.

(24) Buchanan, E. G.; James, W. H.; Gutberlet, A.; Dean, J. C.; Guo, L.; Gellman, S. H.; Zwier, T. S. Single-Conformation Spectroscopy and Population Analysis of Model $\gamma$-Peptides: New Tests of Amide Stacking. Faraday Discuss. 2011, 150, 209-226.

(25) Császár, A. G. Anharmonic Molecular Force Fields. Wiley Interdiscip. Rev. Comput. Mol. Sci. 2012, 2 (2), 273-289.

(26) Laane, J.; Lord, R. C. Far-Infrared Spectra of Ring Compounds. II. The Spectrum and Ring-Puckering Potential Function of Cyclopentene. J. Chem. Phys. 1967, 47 (12), 4941-4945.

(27) Mahé, J.; Jaeqx, S.; Rijs, A. M.; Gaigeot, M.-P. Can Far-IR Action Spectroscopy Combined with BOMD Simulations Be Conformation Selective? Phys. Chem. Chem. Phys. 2015, 17, 2590525914.

(28) Yatsyna, V.; Bakker, D. J.; Feifel, R.; Rijs, A. M.; Zhaunerchyk, V. Aminophenol Isomers Unraveled by Conformer-Specific Far-IR Action Spectroscopy. Phys. Chem. Chem. Phys. 2016, 18 (8), 62756283.

(29) Bakker, D. J.; Peters, A.; Yatsyna, V.; Zhaunerchyk, V.; Rijs, A. M. Far-Infrared Signatures of Hydrogen Bonding in Phenol Derivatives. J. Phys. Chem. Lett. 2016, 7 (7), 1238-1243.

(30) Pirali, O.; Vervloet, A. M.; Mulas, G.; Malloci, G.; Joblin, C. High-Resolution Infrared Absorption Spectroscopy of Thermally Excited Naphthalene. Measurements and Calculations of Anharmonic Parameters and Vibrational Interactions. Phys. Chem. Chem. Phys. 2009, 11, 3443-3454.

(31) Rijs, A. M.; Kay, E. R.; Leigh, D. A.; Buma, W. J. IR Spectroscopy on Jet-Cooled Isolated Two-Station Rotaxanes. J. Phys. Chem. A 2011, 115 (34), 9669-9675.

(32) Frisch, M. J. G.; Trucks, W.; Schlegel, H. B.; Scuseria, G. E.; Robb, M. A.; Cheeseman, J. R.; Scalmani, G.; Barone, V.; Mennucci, B.; Petersson, G. A.; et al. Gaussian 16, rev. A.03; Gaussian, Inc.: Wallingford, CT, 2016.

(33) Becke, A. D. Density-functional Thermochemistry. III. The Role of Exact Exchange. J. Chem. Phys. 1993, 98 (7), 5648-5652. 